\title{
Synthèse des observations d'érosion-corrosion recueillies en centrale sur des circuits en vapeur humide
}

\author{
Summary of erosion-corrosion observations \\ made in power stations on damp-steam circuits
}

\author{
L. Lacaille \\ EDF - Direction des Études et Recherches
}

Phénomènes d'érosion-corrosion, observés dans différentes centrales PWR.

Pour analyser les phénomènes d'érosion-corrosion, rechercher les paramètres influents, en évaluer l'importance relative, un groupe de travail EDF et AlsthomAtlantique a suivi systématiquement plusieurs centrales PWR, considérées comme prototypes. En fonction de ces observations, des essais et expériences ont été réalisés pour peser sur ces paramètres influents avec la collaboration des personnels d'exploitation de ces centrales.

\section{Centrale de CHOOZ}

Une tranche de $300 \mathrm{MW}$ couplée en 1967.

Les tuyauteries de liaison entre corps HP et sécheurs sont en acier au carbone, mais équipées de coudes droits ailetés.

Aprés 36000 heures de fonctionnement, on notait la destruction des ailettes en acier ordinaire des coudes et des attaques à la sortie de ces derniers $(\Delta \mathrm{e}=3 \mathrm{~mm})$. Malgré une augmentation de la puissance de la tranche, les attaques devaient se ralentir après le remplacement des coudes et leurs ailettes réalisés en acier austénitique ( $\Delta$ e en aval : 0,2 à $0,3 \mathrm{~mm}$ ).

Depuis 1978 , le conditionnement du circuit secondaire est assuré à l'aide de morpholine pour obtenir un $\mathrm{pH}$ de $9,1-9,2$ à $25^{\circ} \mathrm{C}$ dans l'eau alimentaire des GV. Une bâche alimentaire dégazante permet d'éviter l'injection d'hydrazine $\left(\mathrm{O}_{2}<1 \mu \mathrm{g} / \ell\right.$ à la sortie du dégazeur).

Après 70000 heures de fonctionnement, l'érosioncorrosion ne concernait plus que de très faibles surfaces en amont des séparateurs-surchauffeurs, mettant en évidence le rôle bénéfique de la morpholine comme réactif de conditionnement.

\section{Centrale de TIHANGE I}

Deux groupes de $510 \mathrm{MW}$, couplés en mars 1975. Leur conception et leur technologie sont très proches de celles des groupes de $900 \mathrm{MW}$. Aussi le suivi du comportement des matériels de cette centrale revêtait-il une importance particulière.

\section{a) Conditionnement chimique.}

Pendant 19700 heures, conditionnement du circuit secondaire par injection d'hydrazine à l'aval des pompes d'extraction, pour obtenir un $\mathrm{pH}$ de 9,1-9,2 dans l'eau alimentaire. En fait, conditionnement ammoniacal de la sortie des GV au condenseur par décomposition thermique totale de l'hydrazine dans les générateurs de vapeur. En raison des destructions importantes constatées dans la turbine HP, les conduites de liaison et les sécheurs-surchauffeurs, ce type de conditionnement a été abandonné.

A partir de 19700 heures de fonctionnement, la morpholine a été adoptée comme seul róactif de conditionnement avec un pH recherché de 9,10-9,15 dans l'eau alimentaire. L'érosion-corrosion a alors régressé d'une manière spectaculaire. C'est ainsi que les teneurs en fer total ont été diminuées:

- d'un facteur 3 dans l'eau alimentaire et purges GV ; - d'un facteur 11 dans les purges des séparateurs; - d'un facteur 5 dans les bâches de récupération des purges.

b) Tenue de toutes les parties en acier $13 \mathrm{Cr}$ Mo 44 (chromesco $1: 1 \% \mathrm{Cr}, 0,5 \% \mathrm{Mo}$ )

Du corps HP aux séparateurs-surchauffeurs, coudes non ailetés et partage du flux de vapeur à l'entrée des sécheurs, réalisés en $13 \mathrm{Cr}$ Mo 44, ont bien résisté à 
l'érosion-corrosion, y compris avec un conditionnement ammoniacal défavorable.

\section{c) Tenue des aciers ordinaires lors du conditionnement ammoniacal}

$35 \%$ des surfaces des tronçons droits des conduites de vapeur humide ont été attaquées par érosioncorrosion $(\Delta \mathrm{e}=2,4$ à $2,7 \mathrm{~mm} / 10000 \mathrm{~h}$ localement) alors que les autres surfaces continuent à être recouvertes de magnétite protectrice.

La destruction par érosion-corrosion concerne plus particulièrement les zônes à forte turbulence de vapeur :

- immédiatement en aval de l'échappement HP ou des coudes, dues aux mauvaises conditions d'écoulement ; - au voisinage des raccordements de soutirage de vapeur lorsque les angles de départs de soutirage sont proches de $90^{\circ}$ alors que ces attaques disparaissent presque totalement lorsque l'angle de raccordement est incliné dans le sens du flux de vapeur principal ;

- dans les zônes avec obstacles (cannes pyrométriques, bourrelets de soudure. . .) ;

- sur la grille de répartition de vapeur à l'amont des sécheurs, exposée frontalement au flux de vapeur humide ( $\Delta \mathrm{e}$ atteint $2,6 \mathrm{~mm}$ en 7500 heures) ;

- l'enveloppe de protection de l'enceinte des sécheurs. En quelques rares endroits $\Delta \mathrm{e}$ atteint $3,1 \mathrm{~mm}$ en 25000 heures.

d) Cas particuliers des surfaces rechargées en $Z 2 C N$ 24-12

L'expérience a montré que le rechargement par un acier fortement allié des zônes particulièrement touchées par l'érosion-corrosion, s'il convenait dans le cas d'érosion pure, n'était pas satisfaisant lorsqu'il s'agissait d'un processus électro-chimique d'érosion-corrosion. En effet, à la périphérie des rechargements, on crée un couplage de métaux avec formation en milieu aqueux d'une pile dont la différence de potentiel est non négligeable (400 $\mathrm{mV}$ environ). Elle entraine une érosioncorrosion plus rapide de l'acier au carbone à la périphérie du rechargement.

\section{e) Tenue des aciers ordinaires lors du conditionnement morpholine}

De nombreuses surfaces, antérieurement attaquées, ont été repassivées avec formation d'une nouvelle couche de magnétite. D'autres surfaces, non repassivées, n’ont subi aucune perte de métal, le $\mathrm{pH}$ du film liquide sur la paroi étant encore insuffisant $(9,1)$ pour permettre la formation d'une couche de magnétite dans ces zônes de fortes turbulences.

\section{Centrale de Doel}

Deux tranches de $417 \mathrm{MW}$, mises en service en 1974 et 1975 .

Les postes d'eau sont équipés de dispositifs de déminéralisation totale de l'eau d'extraction. Conditionnement ammoniacal afin d'obtenir un $\mathrm{pH}$ de 9 dans l'eau alimentaire.

Les conduites de vapeur humide sont en acier E 36 mais équipées de coudes ailetés, à $14 \%$ de chrome, de géométrie soignée. Les attaques par érosion-corrosion n'ont pas dépassé, très localement, $0,4 \mathrm{~mm}$.

Les tuyauteries de soutirage de vapeur, en amont des séparateurs surchauffeurs sont plus attaquées ( $\Delta \mathrm{e}=4 \mathrm{~mm}$ après 23000 heures).

Dans les viroles d'entrée des ballons sécheurssurchauffeurs, $\Delta \mathrm{e}$ atteint $2,4 \mathrm{~mm}$ à $23000 \mathrm{~h}$. Dans la partie inférieure de ces appareils, on observe des dépôts abondants d'oxyde de fer contenant des sels solubles $\left(\mathrm{Cl}^{-}=160 \mathrm{ppm}\right)$.

La présence de coudes ailetés dans les conduites de vapeur ainsi que l'influence du poste de déminéralisation totale sont des facteurs favorables à la limitation de l'érosion-corrosion.

\section{Centrale de Fessenheim}

Deux tranches de $900 \mathrm{MW}$ mises en service en 1977.

Cette centrale a présenté un grand intérêt puisque pendant le premier cycle de fonctionnement, le circuit secondaire de la tranche I a été conditionné à la morholine pendant une période assez longue, la tranche II étant maintenue à un conditionnement ammoniacal.

\section{a) Comportement de la tranche I (conditionnement essentiellement morpholine)}

Du corps HP aux séparateurs-surchauffeurs, des tronçons entiers de conduites de vapeur humide, en acier au carbone, sont intacts. Sur d'autres, les attaques sont relativement faibles. Les zônes affectées paraissent en "voie de guérison". L'observation minutieuse permet de distinguer:

- des zônes comportant une couche de magnétite protectrice représentant la plus grande partie des surfaces ;

- des zônes ayant perdu de l'épaisseur lors du conditionnement ammoniacal et partiellement repassivées. La couche de magnétite est déjà résistante ;

- des zônes en cours de repassivation, dont le faciès gris-noir est totalement différent de celui rencontré dans la phase active d'érosion-corrosion.

\section{b) Comportement de la tranche II (conditionnement ammoniacal}

Contrairement à la tranche I, l'érosion-corrosion y est spectaculaire. Des rechargements on dû être effectués sur certaines parties du corps HP et sur ses diaphragmes.

Des tronçons droits, depuis l'échappement HP jusqu'au dernier coude avant les séparateurs-surchauffeurs, sont attaqués sur $30 \%$ de leur surface. Les pertes d'épaisseur varient de 0,6 à $1 \mathrm{~mm} / 10000 \mathrm{~h}$.

A l'aval des coudes, en $13 \mathrm{Cr}$ Mo 44, les phénomènes d'érosion-corrosion sont intenses, en raison probablement du couplage de métaux à potentiels différents $(\Delta \mathrm{e}: 0,9$ à $2 \mathrm{~mm}$ en $10000 \mathrm{~h})$. Il en est de même en aval de coudes laminaires et pièges à eau réalisés en acier austénitique. 
Le comportement différent de ces deux tranches met en évidence le rôle bénéfique du conditionnement morpholine déjà observé à Chooz et Tihange.

\section{Observations sur le comportement en vapeur humide de différentes nuances d'acier}

Des éprouvettes ont été placées à l'intérieur des conduites de vapeur humide, entre corps HP et sécheurs-surchauffeurs, dans les centrales de Chooz, Doel, Fessenheim, Tihange. Elles ont été installées au voisinage des parois, exposées frontalement au flux de vapeur, conditions les plus sévères auxquelles les aciers de cette partie du circuit secondaire ne sont généralement pas confrontés. Les vitesses de vapeur sont comprises entre $40 \mathrm{~m} / \mathrm{s}$. et $50 \mathrm{~m} / \mathrm{s}$, les nuances d'acier testées ont été choisies dans une gamme allant de l'acier ordinaire aux aciers austénitiques à forte teneur en chrome. Les éprouvettes ont fait l'objet d'observations régulières. Les plus anciennes d'entre elles totalisent $40000 \mathrm{~h}$ de fonctionnement,

\section{Aciers à teneur en chrome $\geqslant 2 \%$}

Quel que soit le traitement chimique de conditionnement de la vapeur, les aciers du type Z2CN 18-10, Z6CNT 18-11, Z5CDA 12, EM 12, 10 CD 9-10, APS $10 \mathrm{M} \mathrm{4}$, sont insensibles aux phénomènes d'érosioncorrosion.

\section{Acier à très faible teneur en chrome $(C r \approx 1 \%)$}

Ces aciers du type $13 \mathrm{Cr}$ Mo 44 (chromesco 1) ou Corten $\mathrm{B}(\mathrm{Cu} \approx 0,4 \%)$ paraissent à la limite de l'apparition des phénomènes d'érosion-corrosion. Les pertes d'épaisseur, très faibles, n'ont été mesurables qu'après $17000 \mathrm{~h}$ de fonctionnement à Tihange : $0,06 \mathrm{~mm}$ pour $13 \mathrm{Cr}$ Mo 44 et $0,15 \mathrm{~mm}$ pour Corten B. Les attaques ont été ensuite arrêtées dès le passage du conditionnement ammoniacal au conditionnement morpholine.

A noter toutefois la très bonne tenue de tous les coudes des tuyauteries de liaison en $13 \mathrm{Cr}$ Mo 44, même en conditionnement ammoniacal, ces coudes étant exposés moins sévèrement que les éprouvettes d'essai.

\section{Aciers ordinaires (type E 26-2)}

L'acier ordinaire est très sensible à l'érosioncorrosion, plus particulièrement lorsque le circuit secondaire est conditionné à l'ammoniaque.

Dès $3000 \mathrm{~h}$, on note l'apparition d'un faciès "en peau d'orange" caractéristique d'une érosion-corrosion continue. Les pertes d'épaisseur, à Tihange, sont de l'ordre de $5 \mathrm{~mm} / 10000 \mathrm{~h}$ au voisinage de la paroi, en raison de l'humidité plus importante dans cette zône.

Dès le traitement à la morpholine, avec un pH limité à 9,1 dans l'eau alimentaire, réduction très nette des pertes d'épaisseur observées à Tihange dans les conditions très sévères d'exposition au flux de vapeur humide $(\approx 1 \mathrm{~mm} / 10000 \mathrm{~h}$, au voisinage de la paroi). Dans les conditions normales d'exposition, ces aciers ordinaires, avec un conditionnement chimique identique sont peu ou pas attaqués par érosion-corrosion.

Des protections de l'acier ordinaire par projection ont été testées sur certaines éprouvettes. Elles se sont avérées totalement inefficaces et ont disparu à moins de $3000 \mathrm{~h}$ de fonctionnement.

\section{Erosion-corrosion observée en fonction de la qualité de l'écoulement}

\section{Rôle des coudes}

\section{a) Coudes normaux}

L'aval des coudes est toujours une zône d'attaque importante d'érosion-corrosion, en raison d'une part des turbulences aérodynamiques et d'autre part de la centrifugation des gouttes et des projections d'eau dues aux décollements du film liquide. Ainsi, à Tihange, en période de conditionnement ammoniacal, l'érosioncorrosion des aciers au carbone, est deux fois plus importante à la sortie des coudes que dans les parties droites plus éloignées.

\section{b) Coudes ailetés}

La mise en place de coudes ailetés est un élément favorable, grâce aux améliorations apportées à l'écoulement et à l'effet de masque des ailettes qui captent une partie importante de l'eau.

\section{c) Coudes "laminaires"}

La recherche d'une réduction des attaques par érosioncorrosion a conduit à l'expérimentation de coudes "laminaires", à section circulaire ou elliptique, divergents-convergents. Leur géométrie permet de maintenir une pression constante le long de l'intrados afin d'y réduire les décollements. Leur section peut être elliptique et cela pour conserver une courbure proche de celle d'un coude normal (profils identiques dans le plan de symétrie).

Des essais sur maquette en air avec pulvérisation d'eau ont permis d'observer un comportement très différent de la phase liquide dans le cas d'un coude normal (jet central en sortie) et d'un coude laminaire (deux lames d'eau longeant les parois à l'aval).

Leur efficacité est liée à celle de pièges à eau suscep. tibles de capter ces lames d'eau et discutée plus loin.

\section{Influence des soutirages}

Les zones voisines des raccordements de tuyauteries de soutirage ou d'équilibrage sont soumises à de fortes destructions, en raison des perturbations dans l'écoulement de la vapeur et de la phase liquide. En conditionnement ammoniacal, les pertes d'épaisseur peuvent être multipliées d'un facteur 4 en aval de ces soutirages et cela pour des angles de raccordement voisins de $90^{\circ}$. Les attaques diminuent fortement avec l'angle de raccordement et s'annulent pratiquement lorsque celui-ci atteint $45^{\circ}$. 


\section{Sécheurs-surchauffeurs}

L'entrée de ces appareils constitue une zône où les champs de vitesse sont particulièrement perturbés : décollements, survitesses locales élevées, fortes composantes tourbillonnaires. . sont des facteurs qui aggravent considérablement les phénomènes d'érosion-corrosion.

Des essais sur maquette hydraulique ont permis de tester des dispositions destinées à améliorer l'écoulement dans les sécheurs-surchauffeurs. Elles ont été adoptées pour la plupart des Centrales EDF du palier $900 \mathrm{MW}$. Elles se traduisent par une meilleure tenue des appareils à l'érosion-corrosion et devraient également s'accompagner d'une efficacité accrue des éléments sécheurs mieux alimentés.

Ōbservations sur l'influence de l'humidité dans les zones affectées par l'érosion-corrosion

\section{Influence du taux de primage à la sortie des générateurs de vapeur}

Le taux de primage à la sortie des générateurs de vapeur modifie très faiblement l'humidité en sortie de corps HP et la morphologie de la phase liquide. L'influence de ce paramètre est d'ordre purement chimique par la présence en quantité légèrement supérieure de composants entraînés par les gouttes, avec une légère incidence sur le milieu électrolytique au voisinage des parois exposées à la vapeur humide. Les observations dans différentes centrales n'ont pu mettre en évidence l'influence du taux de primage sur l'érosion-corrosion.

\section{Essais de réduction de lhumidité par l'installation de pièges à eau à l'échappement'HP}

L'humidité de la vapeur ayant un rôle déterminant dans les attaques par érosion-corrosion, Alsthom-Atlantique a cherché à les réduire en réalisant une pré-séparation de la phase liquide à l'aide d'appareils expérimentaux, type piège à eau. Les essais réalisés à Fessenheim et Tihange ont montré qu'ils ne constituaient pas une protection suffisante contre l'érosion-corrosion.

Certes, $30 \%$ de l'eau sont récupérés immédiatement en aval de ces appareils, mais l'existence d'un film liquide légèrement réduit ne peut empêcher le processus électrochimique du phénomène d'érosion-corrosion dans un milieu électrolytique non supprimé.

Solution d'avenir aux problèmes d'érosion corrosion : les sécheurs à grande vitesse (SGV).

La suppression totale de l'humidité dès l'échappement HP nous mettrait à l'abri des phénomènes d'érosion- corrosion dans les Centrales PWR, quels que soient les aciers et modes de conditionnement choisis, nous replaçant dans les conditions classiques des turbines à vapeur des centrales fossiles.

La Direction des Etudes et Recherches d'Electricité de France a mis au point des appareils susceptibles d'atteindre cet objectif. Les séparateurs à grande vitesse sont un type particulier de séparateurs centrifuges, constitués de cellules de séparation placées en parallèle dans les tuyauteries de liaison, immédiatement à l'échappement du corps HP de la turbine.

La quasi-totalité de l'eau est ainsi captée et son évacuation favorisée à l'aide d'un prélèvement de vapeur en mettant à profit, par exemple, le soutirage de vapeur prévu généralement à la sortie du corps HP.

Des essais ont été effectués sur une première génération de SGV. Les humidités atteintes à la sortie de ces appareils ont été inférieures à $0,1 \%$. Deux unités de démonstration sont installées depuis 1980, sur la tranche II du Bugey et leur développement industriel est assuré dans le cadre d'un contrat de collaboration passé avec Alsthom-Atlantique et Stein-Industrie.

Si les résultats d'essais actuels étaient confirmés, les avantages de ces appareils seraient indéniables :

- suppression totale de tout problème d'érosioncorrosion dès l'échappement de la turbine HP ;

- possibilité de réalisation des tuyauteries de liaison en acier ordinaire quel que soit le traitement utilisé pour le conditionnement chimique de la vapeur ;

- réduction très importante de la taille des séparateurs devant permettre d'abaisser le coût propre des sécheurssurchauffeurs actuels et, plus généralement, celui de la salle des machines.

\section{Conclusions}

L'observation pendant plusieurs années de cinq grandes centrales à eau légère permet de dégager un certain nombre de solutions à l'érosion-corrosion.

Les aciers dont la teneur en chrome est de $2 \%$ sont insensibles aux phénomènes d'érosion-corrosion en vapeur humide. Les aciers ordinaires, ou à très faible teneur en chrome $(1 \%)$ peuvent résister à l'érosioncorrosion, à condition de maintenir, dans la phase liquide, un $\mathrm{pH}$ suffisant. A cet effet, la morpholine, grâce à son faible coefficient de partage, est un excellent réactif de conditionnement.

La possibilité d'installer des Sécheurs à Grande Vitesse en sortie de corps HP est une solution d'avenir. 


\section{Abstract}

Summary of erosion-corrosion observations made in power stations on damp-steam circuits

From the HP turbine up to and including the separator feed heaters, there has been considerable erosion-corrosion of carbon steels in the PWR stations under observation; it has affected about $35 \%$ of surfaces with losses of thickness locally attaining $2.7 \mathrm{~mm} / 10,000 \mathrm{~h}$.

This erosion-corrosion has been stopped at Chooz by morpholine treatment of the secondary circuit together with the use of finned elbow pieces.

At Tihange, treatment with morpholine has caused a spectacular reduction in erosion-corrosion. Compared with ammonia treatment, total content of iron in the drain water from the separators has been reduced by a factor of 11 ; this value is strongly representative of erosion-corrosion phenomena.

At Doel, despite the use of ammonia treatment, erosioncorrosion has been limited by the total demineralization unit and the use of finned elbow joints.

At Fessenheim, one unit has been treated with ammonia and the other with morpholine. The former showed extensive destruction by erosion-corrosion, while in the latter there was repassivation after the adoption of morpholine as the treatment reagent.

Samples of different grades of steel were placed under severe exposure conditions facing a flow of damp steam in various PWR stations. Useful information was obtained after 40,000 hours of test:

- steels with low chromium content $(\mathrm{Cr}=1 \%)$ appear to be on the threshold of the appearance of erosion-corrosion phenomena, though these steels stand up well when used for elbow joints;

- steels containing about $2 \%$ chromium are unaffected by erosion-corrosion and make it possible to avoid the use of austenitic steels, which are subject to corrosion under tension.

While finned elbow joints improve the flow, the experiment with "laminar" elbows, of circulat or elliptic section, divergentconvergent, did not give the expected results.

An attempt was made to reduce the humidity of the steam at the HP output by preseparation of the liquid phase using experimental equipment of the water-trap type. While $30 \%$ of the water is recovered with this equipment, the slight reduction in the liquid film cannot prevent the electrochemical process of erosion-corrosion in a unsuppressed electrolytic environment.

High-speed driers seem to be a solution to the problems of erosion-corrosion for the future in PWR stations; they would result in total elimination of humidity in the HP exhaust, and consequently remove all problems of erosion-corrosion, whatever steels or treatments are used.

The Studies and Research Division of Electricité de France has developed such devices: they are a special type of centifugal separator, consisting of separation cells placed in parallel in the linkage piping immediately after the HP turbine.

Two experimental units have been installed since 1980 in the Le Bugey station. The humidities attained at the output from them are less than $0.1 \%$, thus bringing us back to the classical conditions in the steam turbines of fossil-fuel power stations, and eliminating all erosion-corrosion problems.

If the test results were confirmed, the very substantial reduction in the size of the separators should make it possible to lower the cost of the drier-feed heaters, and in general that of the machine room. 


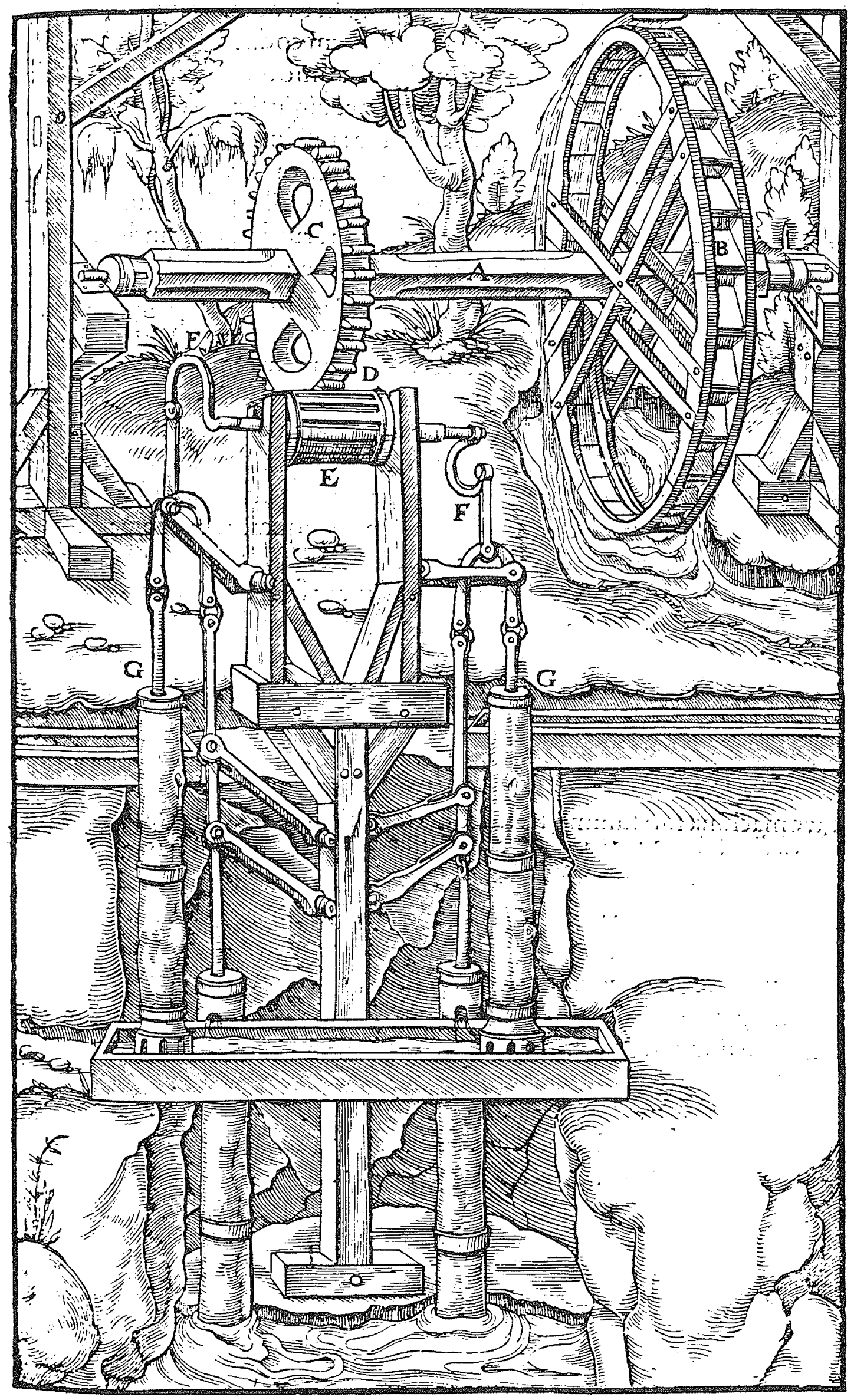

